\title{
1999: ET's fifteenth year
}

When the English Today project was proposed to me in the early ' 80 s by a most prescient and innovative publisher, Adrian du Plessis, I was already committed to and curious about English in all its forms, but at the time my career was focused on ESL in French Canada, at the linguistically highly politicized Université du Québec à Trois-Rivières. Academics were well paid in Canada in those days, and it was hard to consider the loss of income entailed if I re-traversed the Atlantic to head a project that might not even get off the ground. And I would have to get it to fly while making up the rest of what a family of five needed through writing, consultancy, and the like. Madness, many said, but it is a madness I have had no cause to regret.

At first ET was a glossy mag that made a media splash. It did well as a consumer periodical, but both Cambridge University Press and librarians had problems with it, so it in due course morphed into the format and style we still maintain, and gradually became more academic. But even now the question of whether ET is 'properly' a scholarly journal or is a one-off popular-cum-academic hybrid is still being discussed. And may it continue to be so discussed.

The 'hybrid' concept has certainly evolved strongly and well: a medium that allows for rigorous and relevant scholarship that is also accessible and compact. ET's pages are filled with detail, data and debate frae aa the airts the wind can blaw. But there is plenty still to be done, especially in encouraging the wider circulation and discussion of information and ideas about the intriguing yet unnerving language on which we focus. Among the sustaining satisfactions for all involved is the high level of original research and reflection - and often the intellectual daring - of our contributors, endorsed so notably by the many citations of their work in articles, papers, and books about English wherever one looks. In our fifteenth year we can celebrate a tradition of solid, often ground-breaking reporting and a marked influence - and take them further.

Tom McArthur

The editorial policy of Eng/ish Today is to provide a focus or forum for all sorts of news and opinion from around the world. The points of view of individual writers are as a consequence their own, and do not reflect the opinion of the editorial board. In addition, wherever feasible, ET generally leaves unchanged the orthography (normally British or American) and the usage of individual contributors, although the editorial style of the journal itself is that of Cambridge University Press. 